\title{
Potential combined pro-cognitive, anxiolytic and antidepressant properties of novel GABAA receptor positive modulators with preferential efficacy at the a5- subunit
}

Thomas D. Prevot ${ }^{1}$, Guanguan $\mathrm{Li}^{2}$, Aleksandra Vidojevic ${ }^{3}$, Keith A. Misquitta ${ }^{1,4}$, Corey Fee ${ }^{1,4}$ Anja Santrac ${ }^{3}$, Daniel E. Knutson², Michael R. Stephen², Revathi Kodali², Nicolas M. Zahn², Leggy A. Arnold ${ }^{2}$, Petra Scholze ${ }^{5}$, Janet L. Fisher ${ }^{6}$, Bojan D. Marković ${ }^{7}$, Mounira Banasr ${ }^{1,4,8}$, Jim Cook $^{2}$, Miroslav Savic ${ }^{3 *}$ and Etienne Sibille ${ }^{1,4,8 *}$.

${ }^{1}$ Campbell Family Mental Health Research Institute of CAMH, Toronto, Canada

${ }^{2}$ Department of Chemistry and Biochemistry, University of Wisconsin-Milwaukee, Milwaukee,

${ }^{3}$ Department of Pharmacology, Faculty of Pharmacy, University of Belgrade, Vojvode Stepe 450, 11221 Belgrade, Serbia

${ }^{4}$ Department of Pharmacology and Toxicology, University of Toronto, Toronto, Canada

${ }^{5}$ Department of Pathobiology of the Nervous System, Center for Brain Research, Medical University of Vienna, Vienna, Austria

${ }^{6}$ Department of Pharmacology, Physiology and Neuroscience, University of South Carolina School of Medicine, Columbia South Carolina USA

7 Department of Pharmaceutical Pharmacy, Faculty of Pharmacy, University of Belgrade, Vojvode Stepe 450, 11221 Belgrade, Serbia

${ }^{8}$ Department of Psychiatry, University of Toronto, Toronto, Canada

${ }^{*}$ Corresponding authors

Etienne Sibille, Ph.D., CAMH, 250 College street, room 134, Toronto, ON M5T 1R8, Canada Tel: 416-535-8501, ext 36571; E-mail: Etienne.sibille@camh.ca Miroslav Savic, Ph.D. Faculty of Pharmacy, University of Belgrade, Vojvode Stepe 450, 11221 Belgrade. Tel: +3816427551447; E-mail: miroslav@pharmacy.bg.ac.rs

Short title: Combined properties on mood and cognition of new a5PAM

Abstract: 242 words

Main text: 4026 words

Number of figures: 5

Number of supplementary information: 14 methods, 12 tables and 6 figures 


\section{ABSTRACT}

Altered $\mathrm{y}$-aminobutyric acid (GABA) function is consistently reported in psychiatric disorders, normal aging and neurodegenerative disorders, and reduced function of somatostatin expressing GABA interneurons is associated with both mood and cognitive symptoms. Somatostatin-neurons signal in part through $\alpha 5$-subunit containing GABA $_{A}$ receptors ( $\alpha 5$ GABAA-Rs) which are localized in brain regions implicated in emotion and cognition. We hypothesize that enhancing a5-GABAA-R activity has therapeutic potential for both mood and cognitive symptoms in stress-based and aging rodent models.

We synthesized four novel imidazobenzodiazepine (IBZD) amide ligands, tested them for positive allosteric modulation at a5-GABAA-R ( $\alpha 5-\mathrm{PAM})$, pharmacokinetic properties, and for anxiolytic and antidepressant activities in adult mice. Pro-cognitive activity was tested in adult mice submitted to chronic stress and in old mice. Diazepam (DZP), with broad PAM activity at GABAA-Rs, was used as a control.

Three novel IBZD amide ligands (GL-II-73, GL-II-74 and GL-II-75) demonstrated adequate brain penetration, affinity and a5-PAM activity, and metabolic stability for in vivo studies. GL-II73/74/75 showed significant anxiolytic and antidepressant efficacies in adult mice. GL-II-73 and GL-II-75 significantly reversed cognitive deficits induced by stress or occurring throughout normal aging. This activity was maintained after sub-chronic administration for GL-II-73. In contrast DZP displayed anxiolytic but no antidepressant or pro-cognitive activities.

We demonstrate for the first time the potential for combined anxiolytic, antidepressant and procognitive therapeutic, mediated by newly designed IBDZ amide ligands with efficacy at a5GABAA-Rs. These results suggest a novel therapeutic approach targeting both mood and cognitive symptoms in depression and/or aging. 


\section{INTRODUCTION}

Altered levels and signaling of gamma-aminobutyric acid (GABA), the major inhibitory neurotransmitter, is frequently reported in multiple psychiatric disorders (depression $(1,2)$, schizophrenia (3), anxiety disorder (4)), normal ageing $(5,6)$ and neurodegenerative disorders (Alzheimer's disease, AD) $(6,7)$ in multiple brain regions including the prefrontal cortex (8), anterior cingulate cortex (9) and amygdala (10). Postmortem studies have associated these GABAergic deficits to a reduction in number and/or loss of function of somatostatin-expressing GABA interneurons in multiple brain regions (see (6) for review). Previous studies showed that impaired inhibition from somatostatin-expressing GABA neurons mediated emotionality (11-13) and cognitive deficits (14-16).

GABA receptors are represented by two classes: $G_{A B A}$ and $G_{A B} A_{B}$ receptors. $G_{A B A}$ receptors (GABAA-Rs) are composed of multiple subunits $(\alpha, \beta, \gamma, \delta, \varepsilon, \theta, \pi$ and $\rho)$ assembled into pentamers (17) forming a chloride channel. GABA released from somatostatin interneurons are thought to signal, in large part, through the a5-containing GABAA-Rs, due to their preferential localization to extrasynaptic distal dendritic regions regulating tonic inhibition (1821), and additionally through the ubiquitously-expressed a1-GABAA-Rs. Benzodiazepines (BZs; such as diazepam, DZP) and imidazobenzodiazepines (IBZDs, such as flumazenil) represent the well-established classes of therapeutics acting on GABAA-Rs. BZs are prescribed to alleviate the burden of anxiety (22), but have debatable efficacy on the core symptoms of depression such as anhedonia (23). BZs modulate GABAA-R activity as non-selective positive allosteric modulators (PAMs), via binding between the $\mathrm{y} 2$ and either the $\alpha 1, \alpha 2$, $\alpha 3$ or $\alpha 5$ subunit (24). This broad receptor activity contributes to significant side-effects (sedation, hypnosis, ataxia, addiction, amnesia), limiting their therapeutic potential (25). However, the relations are not straightforward; for instance, high activity at a1-GABAA-Rs induces sedation and contributes to amnesia, but the same receptor subtype is also proposed to be implicated in cognition (26). The BZ anxiolytic properties are mediated predominantly by a2-GABAA-Rs (27). 
a5-GABAA-Rs have predominant distribution in the neocortex and hippocampus (28) suggesting a role in cognition and emotion (29,30). As a5-PAMs can alleviate behavioral emotionality in a mouse model of schizophrenia $(31,32)$ and depression $(33)$, or exert procognitive efficacy in old rats (34), we hypothesize that bypassing the somatostatin/GABA interneurons deficiency through enhanced a5-GABAA-R activity may represent a novel target for combined therapeutic actions on mood and cognition.

Based on an IBZD structure (a BZ-imidazol hybrid) we developed ethyl esters of IBZDs with a C(4) R-CH3 substituent that bind preferentially to the a5 $32 / 3 \gamma 2$ GABAA-Rs (35-37) and that are active in models targeting schizophrenia (31,32) and depression (33) but quickly metabolized (38). Because of their improved metabolic stability and bioavailability as compared to esters, amides are commonly-used replacements (39). Hence, a series of amides were designed that fit the pharmacophore/receptor model (37), with increased binding affinity and efficacy at a5-GABAA-Rs, compared to corresponding esters (40). We describe here the synthesis, pharmacokinetic, functional and behavioral characteristics of four IBZD amide ligands and we predicted that the novel a5-PAMs will have significant antidepressant potential and procognitive activity in adult and old mice, with limited adverse effects. 


\section{METHODS AND MATERIALS}

Detailed methods are provided in the supplements.

\section{Chemistry}

Novel IBZD amide analogs were synthesized based on the structures of the existing ligands relatively selective for a5-GABAA-Rs, i.e. SH-053-2'F-R-CH3 and MP-III-022 (41). These new ligands contain a chiral (R) C(4)-methyl group and a 2'F pendent phenyl ring, as general structural requirements for improved selectivity at the a5-subtype based on the pharmacophore model (37). All four amide analogs, referred herein as GL-II-73, GL-II-74, GL-II-75, and GL-II-76, were prepared from the SH-053-2'F-R-CH3 following steps described in the Supplementary Methods and presented in Supplementary Figure S1.

\section{Electrophysiological recordings}

Electrophysiological recordings were performed as described in (42) (Supplementary Methods). Briefly, HEK-293T cells were transiently transfected with mammalian clones of GABAA-Rs. Currents in response to $\mathrm{GABA}$ and $\mathrm{GABA}+$ modulators $\left(\mathrm{EC}_{3-5} \mathrm{GABA}\right)$ were measured using voltage-clamp recordings in the whole-cell configuration.

\section{Binding studies}

Binding studies were performed on human embryonic kidney (HEK) 293 cells following the methods described in (39) (Supplementary Methods). HEK293 cells were transfected with cDNAs encoding rat GABAA-R subunits subcloned into $\mathrm{pCl}$ expression vectors. To determine the equilibrium binding constant $K D$ of $[3 \mathrm{H}]$-flunitrazepam for the various receptor-subtypes, membranes were incubated with various concentrations of $[3 \mathrm{H}]$-flunitrazepam in the absence or presence of 5mM DZP. Drug concentrations resulting in half maximal inhibition of specific [3H]- 
flunitrazepam binding (IC50) were converted to Ki values by using the Cheng-Prusoff relationship (43).

\section{Animals}

Young (2-3 months) or old (21-22 months) C57BL/6 mice were kept in normal housing conditions with a $12 \mathrm{hr}$ light-dark cycle, water and food ad libitum. Prior to behavioral assessment, animals were handled daily for $5 \mathrm{~min}$ to reduce acute anxiety-like responses (44). Testing took place during the light phase and was conducted in accordance with local ethical commission on animal experiment (see supplementary for details). To induce a cognitive deficit in young mice, animals were subjected to a chronic stress protocol (CS). They were placed, twice a day for $1 \mathrm{hr}$, in a $50 \mathrm{ml}$ Falcon $\AA$ tube perforated on each end to let the animal breathe. CS was applied for at least 10 days before testing but was not applied on testing days.

\section{Ligand preparation and administration}

Ligands were diluted in a vehicle solution containing $85 \%$ distilled $\mathrm{H}_{2} \mathrm{O}, 14 \%$ propylene glycol and $1 \%$ Tween-80 to be administered intraperitoneally (i.p.) at a volume of $10 \mathrm{ml} / \mathrm{kg}$. Working solutions were prepared at 1,5 or $10 \mathrm{mg} / \mathrm{kg}$ and adjusted to body weight before injection. DZP was used as a non-selective GABAA-R PAM with known anxiolytic activity, which does not exert known pro-cognitive effects. DZP was administered i.p. at $1.5 \mathrm{mg} / \mathrm{kg}$ based on previous studies (45).

For sub-chronic administration in the drinking water for 10 consecutive days, ligands were diluted in tap-water, stirred overnight at room temperature and provided in glass bottles to tailor for a $30 \mathrm{mg} / \mathrm{kg}$ daily dose. Bottles were changed every other day to provide freshly-prepared solutions.

\section{Pharmacokinetic characterization}


Metabolism studies were performed in human and mouse liver microsomes as described in (46). Briefly, the test ligands were incubated at $10 \mu \mathrm{M}$ with active or heat-inactivated human or mouse liver microsomes and appropriate cofactors. Aliquots were removed at different time points and assayed using a liquid chromatography/tandem mass spectrometry (LC-MS/MS) analytical method. To obtain pharmacokinetic profiles, mice were treated i.p. at $10 \mathrm{mg} / \mathrm{kg}$ and sacrificed at different time points (5-to-720min post-injection) for brain and plasma quantification of ligands by ultraperformance LC-MS/MS (47).

\section{In vitro hydrolytic plasma stability studies}

Each ligand stability was tested in vitro at $37^{\circ} \mathrm{C}$, utilizing blank mouse plasma spiked with the respective ligand and internal standard, as described in (39).

\section{Plasma protein and brain tissue binding studies}

The rapid equilibrium dialysis assay used to determine the free fraction of the ligands in mouse plasma and brain tissue was as described in (47). Free concentrations in the brain were calculated by multiplying the obtained total brain concentrations with the appropriate free fractions determined by rapid equilibrium dialysis.

\section{Behavioral assessment}

The ligands and the non-selective PAM DZP were tested in behavioral tests assessing anxietylike behavior (elevated plus maze, EPM), antidepressant predictability (forced swim test, FST), locomotor activity (open-field), motor coordination (rotarod), and spatial-working memory (Ymaze, YM). See details in the Supplementary methods and Supplementary Figure S2.

\section{Statistical analysis}


All data are expressed as mean \pm SEM. Data obtained from electrophysiology studies are subjected to t-tests comparing the mean to $100 \%$ of response to GABA alone. For behavioral experiments, statistical analyses were performed using one-way ANOVA and post-hoc Scheffe's or Student-Newman-Keuls test when applicable. For the EPM and YM tests, sex was put as a cofactor to investigate potential sex effects on the behavioral outcome. All values obtained after statistical analysis are provided in the Supplementary Tables S1, S2, S8, S9, S10, and S11. 


\section{RESULTS}

\section{Novel IBZD amide ligands potentiate $\alpha$-containing GABAA-Rs}

To assess potentiation at the different $\alpha$-containing recombinant GABAA-Rs (Figure 1a-d and Supplementary Table S1), the effects of four new IBZD amide ligands were compared to the response to GABA alone $\left(\mathrm{EC}_{3-5}\right)$. At $100 \mathrm{nM}$, GL-II-74 and GL-II-75 exhibit substantial allosteric modulation at a5-GABAA-Rs, while GL-II-73 and GL-II-76 exhibited lower, but still significant, potentiation (t-test comparison to $100 \% ; \mathrm{t}>2.9 ; \mathrm{p}<0.04$ ). At this low concentration, all compounds also potentiate $\alpha 1, \alpha 2$ and $\alpha 3-G A B A A-R s ~(t>2.9 ; p<0.04), \quad$ LL-II-75 already exhibiting high potentiation at this subunits, suggesting shared electrophysiological properties with the referent non-selective PAM DZP $(48,49)$. The profiles at $100 \mathrm{nM}$ were confirmed at $1 \mu \mathrm{M}$ concentration, with strong a5-PAM effects (t-test comparison to $100 \%$; $>4.67 ; p<0.009$ ), and still significant potentiation at $\alpha 1, \alpha 2$ and $\alpha 3(t>3.17 ; p<0.03)$. ANOVA comparing potentiations between all subunits for each compound at each concentration revealed that all compounds but GL-II-75 preferentially potentiate a5-GABAA-Rs (ps<0.04; Supplementary Table S2) As expected, $\alpha 4-, \alpha 6-$ or $\delta$-subunit containing GABAA-Rs were not potentiated by the new ligands (Supplementary Table S1) suggesting similar subunit-dependent activity as DZP (48,50-52). Similar potentiation levels were obtained at the $\beta 1$ - and $\beta 3$-containing $\mathrm{GABA}_{\mathrm{A}}$ receptors (besides a significant difference for $\mathrm{GL}-\mathrm{II}-73$ at $1 \mu \mathrm{M}$ concentration $(p=0.03)$, but irrelevant for in vivo application) suggesting no critical impact of the $\beta$-subunit on the modulatory effects of the new ligands (Figure 1e-h and Supplementary Table S3).

\section{Ligands selection and doses validation for animal studies}

Liver microsome metabolism assays (Supplementary Table S4) indicated that GL-II-73 and GL-II-74 had the longest half-lives in mouse and human, respectively, while GL-II-76 had a very short half-life in mice, precluding its use in vivo, hence it was excluded from further analysis. 
Considering the potential implication of the a5-subunit on cognitive functions, and the role of a1-subunit in sedation, we aimed at finding a dose that would potentiate a5-GABAA-Rs preferentially to a1-GABAA-Rs (26). The concentration-response curves for the ligands (Figure 1i-k and Supplementary Table S5) suggest that at $1 \mathrm{mg} / \mathrm{kg}$, expected potentiation would be below $120 \%$ for GL-II-73 and GL-II-75 at both a1- and a5-GABAA-Rs, while GL-II-74 would have significant potentiation at a5-GABAA-Rs. At $10 \mathrm{mg} / \mathrm{kg}$, potentiation would significantly increase for both subunits, with higher potentiation at a5- than a1-GABAA-Rs for GL-II-73 and GL-II-74. Conversely, GL-II-75 exhibits higher potentiation at $\alpha 1$ - than at $\alpha 5-G A B A A-R s$ at $10 \mathrm{mg} / \mathrm{kg}$. Higher potentiation at $\alpha 1$ - than $\alpha 5-G A B A A-R s$ was also observed with the nonselective referent DZP (Figure 1I).

Potentiation of GABA-induced chloride current does not necessarily correlate with binding affinity. Indeed, binding assays at $\alpha 1 / 2 / 3 / 5-G A B A A-R s$ showed that all ligands are affinityselective to the a5-GABAA-R (Ki values being $6-15 x$ more potent at $\alpha 5-G A B A A-R$ than other $\alpha$ GABAARs, Supplementary Table S6). Notably, GL-II-73 showed the lowest affinities, with Ki values in the $\mu \mathrm{M}$ range, compared to the other ligands. In contrast to all four novel ligands, DZP showed high and similar affinities at all $\alpha-$ GABAA-Rs.

Together, these results establish GL-II-73, GL-II-74 and GL-II-75 as ligands with significant PAM efficacies at a5-GABAA-Rs, and low, moderate and higher efficacies at a1/2/3-GABAA-Rs, respectively, and also as a5-GABAA-R-binding preferring ligands, possessing relatively high affinities, with the notable exception of the low $\mu \mathrm{M}$ range for GL-II-73.

\section{Pharmacokinetic, plasma stability and free fraction studies}

Pharmacokinetic profiles of the test ligands and DZP were established in mice after $10 \mathrm{mg} / \mathrm{kg}$ i.p. administration (Figure 2a-d). Plasma and brain free fractions were $20.39 \%$ and $12.14 \%$ for GLII-73, $6.34 \%$ and $4.49 \%$ for GL-II-74, 5.08\% and $1.34 \%$ for GL-II-75, and $16.25 \%$ and $6.38 \%$ for 
DZP, respectively. Elimination half-lives $\left(T_{1 / 2}\right)$ of all three ligands and DZP suggest that DZP and GL-II-74 are the most stable in mouse plasma, while GL-II-73 is most stable in the mouse brain. Maximum brain concentration $\left(\mathrm{C}_{\max }\right)$ was the highest with $\mathrm{GL}-\mathrm{II}-74$, suggesting a relatively optimized capacity for brain targeting. Brain-to-plasma partition coefficient $\left(\mathrm{K}_{\mathrm{p}}\right)$ values showed that GL-II-74 displayed excellent brain permeability, although less efficient than DZP $(K p=1.14$ vs 3.06). This is further supported by the ratio of unbound brain to unbound plasma ligand concentrations values $\left(\mathrm{K}_{\mathrm{p}, \mathrm{uu}}\right)$, a measure of net transport across the blood-brain barrier, which better quantifies the brain penetration efficiency (53). This parameter demonstrates that GL-II73 may be a substrate for efflux transport mechanisms at the blood-brain barrier, compared to GL-II-74 or GL-II-75. Although DZP displayed excellent brain permeability (as measured by $\mathrm{K}_{\mathrm{p}}$ and $\mathrm{K}_{\mathrm{p}, \mathrm{uu}}$ ), GL-II-74 and GL-II-75 reached notably higher AUC brain values (from 0 to $720 \mathrm{~min}$ or infinite time post-dosing) than DZP. The brain AUC value for GL-II-73 was somewhat lower but comparable to DZP.

All three ligands displayed acceptable brain penetration and an excellent in vitro metabolic stability; after $4 \mathrm{~h}$ of incubation in mouse plasma, the fraction of remaining intact ligand was 98.88\%, 85.83\% and 78.52\% for GL-II-73, GL-II-74 and GL-II-75, respectively, whereas the intact fraction of DZP was only $76.36 \%$. Thus, the novel ligands have at least as favorable pharmacokinetic properties as DZP.

The concentrations of all ligands in mouse brain 30 min after single, or $1 \mathrm{~h}$ after the last of three i.p. injections (administered 24h, 20h and $1 \mathrm{~h}$ prior to determination of concentration) at 1 , 5 and $10 \mathrm{mg} / \mathrm{kg}$ (Supplementary Figure S2 and Supplementary Table S7) demonstrated that attainable concentrations were governed by the dose and dosage regimen. In view of the potentiation at $\alpha 1$ - and $\alpha 5-G A B A A-R s$ (Figure 1) and pharmacokinetic properties (Figure 2), doses of 1,5 and $10 \mathrm{mg} / \mathrm{kg}$ doses were chosen for behavioral testing.

\section{Estimated in vivo receptor occupancy}


The relative fraction of receptors occupied at the given free concentration can be approximated from in vitro binding and PK data (Supplementary Table S7). At 10mg/kg, GL-II-73 displayed very low predicted receptor occupancy, including at the a5-GABAA-R (5.95\%). GL-II-75 displayed low values at $\alpha 1 / 2 / 3-$ GABAA-Rs $(<9.31 \%)$, and $38.42 \%$ predicted occupancy at $\alpha 5$ GABAA-Rs. GL-II-74 displayed values in the $17-24 \%$ range for $\alpha 1 / 2 / 3-$ GABAA-Rs and $75.51 \%$ for a5-GABAA-Rs. In contrast, DZP displayed a much greater overall occupancy at all four a5GABAA-R (70\% and above), even at the $1.5 \mathrm{mg} / \mathrm{kg}$ low dose. Despite these variable predicted receptor occupancy levels, we used the electrophysiological responses at a1- and a5-GABAARs as guide for in vivo studies.

\section{Effects of novel IBZD amide ligands with a5-PAM activity on locomotor activity}

The effects of GL-II-73, GL-II-74 and GL-II-75, dosed i.p. at 10mg/kg were assessed on the distance traveled in 5-min bins in the locomotor activity test in mice (Supplementary Figure S3). A two-way repeated-measure ANOVA, followed by post-hoc comparison revealed that GLII-74 induced a hyperlocomotion response in time intervals $0-5 \mathrm{~min}$ and $50-55 \mathrm{~min}$ postinjection, while GL-II-75 elicited a similar stimulant-like effect in the first $5 \mathrm{~min}$, but a consistent hypolocomotion in the $15-40 \mathrm{~min}$ period. DZP dosed at $1.5 \mathrm{mg} / \mathrm{kg}$ i.p. induced a hypolocomotor response in intervals 10-35 $\mathrm{min}$ and 40-55 $\min$ (Supplementary Figure S4), confirming its known sedative effect on spontaneous locomotor activity. At contrary, GL-II-73 had no effect on locomotor activity.

\section{The novel IBDZ amide ligands exhibit anxiolytic and antidepressant-predictive properties}

In the EPM test, all ligands induced a trend or a significant increased percentage of time spent in the open-arms of the EPM (ANOVAs; Fs>3.7; $\mathrm{p}<0.07$ ) Post-hoc analysis identified a significant increase in time after i.p. administration at $10 \mathrm{mg} / \mathrm{kg} \quad(p<0.03)$ for all compounds(Figure 3a-d and Supplementary Table S9). 1.5mg/kg DZP significantly increased 
the time spent in the open-arms $(p=0.02)$, suggesting anxiolytic properties of all ligands, including DZP.

In the FST, GL-II-73, GL-II-74 and GL-II-75 induced a significant decrease in time spent immobile (ANOVA $\mathrm{F}>5.4 ; \mathrm{p}<0.004$ ) compared to the vehicle-injected group after i.p. administration at $10 \mathrm{mg} / \mathrm{kg}(\mathrm{p}<0.003$; Figure $3 \mathrm{e}-\mathrm{h})$. The dose of $5 \mathrm{mg} / \mathrm{kg}$ also reduced the time spent immobile in animals receiving GL-II-74 and GL-II-75 ( $p<0.03)$. In contrast, DZP induced significant increases in time spent immobile $(p=0.004)$, potentially due to locomotor side-effect (Supplementary Figures S5), thus precluding any conclusion as to putative depressant-like effect.

\section{The novel IBZD amide ligands reverse stress-induced working memory deficits}

ANOVAs reveled differences in alternation rate after CS exposure and injection of the ligands $(F>7 ; p<0.0004)$. Young adult mice were exposed to chronic stress (CS) to induce working memory impairments in a YM spatial spontaneous alternation task. CS exposure decreased the alternation rate in animals receiving only vehicle $(p<0.002$; Figures 4A-D and Supplementary Table S9). Administration of GL-II-73 at $10 \mathrm{mg} / \mathrm{kg}$ restored alternation rate in stressed animals to the same level as non-stressed animals, and significantly different from the stressed mice receiving vehicle $(p=0.01)$. $1 \mathrm{mg} / \mathrm{kg}$ and $5 \mathrm{mg} / \mathrm{kg}$ doses were inefficient. In contrast, stressed animals injected with GL-II-74 displayed lower alternation rate than non-stressed animals, suggesting a lack of pro-cognitive effect, regardless of the ligand dose ( $p>0.97)$. GL-II-75 administration restored alternation rate to non-stressed levels at 5 and $10 \mathrm{mg} / \mathrm{kg}(p<0.045)$. As expected, the administration of $1.5 \mathrm{mg} / \mathrm{kg}$ of DZP did not reverse the cognitive deficits induced by $\operatorname{CS}(p=0.94)$.

We also tested the ligands in non-stressed animals to assess putative effects at baseline (Supplementary Figure S6 and Supplementary Table S9). GL-II-73 and GL-II-75 had no effect on alternation rate (ANOVAs $\mathrm{F}<2.4, \mathrm{p}>0.3$ ), whereas $10 \mathrm{mg} / \mathrm{kg}$ of $\mathrm{GL}-\mathrm{II}-74$ and $1.5 \mathrm{mg} / \mathrm{kg}$ 
DZP reduced alternation rates (ANOVAs $\mathrm{F}>8.8$; $\mathrm{p}<0.005$ ), suggesting deleterious effects on working/spatial memory.

\section{GL-II-73 and GL-II-75 reverse age-related working memory deficits}

The pro-cognitive efficacy of GL-II-73 and GL-II-75 was assessed in old male mice. ANOVAs reveled significant differences between young, old and old treated $(F<0.0015)$. 18-month old mice displayed alternation rates in the $\mathrm{Y}$-maze at chance level, suggesting cognitive impairment ( $p<0.002$ compared to young mice). A single $5 \mathrm{mg} / \mathrm{kg}$ administration (i.p) of $\mathrm{GL}-\mathrm{II}-73$ or $\mathrm{GL}-\mathrm{II}-75$ significantly reversed spatial-working memory deficits of old mice to levels indistinguishable from young controls ( $p<0.03$; Figure 4E-F and Supplementary Table S10).

\section{GL-II-73, but not GL-II-75, maintained pro-cognitive activity after sub-chronic administration in young and old mice.}

The two ligands that exhibited pro-cognitive efficacy after a single acute i.p. injection (GL-II-73 and GL-II-75) were tested via sub-chronic administration in drinking water (Figure 5 and Supplementary Table S11). In the adult stress-induced cognitive deficit model, ANOVA analysis revealed significant differences between groups $(F(2,14)=28.1 ; p=0.0001)$ only with GL-II-73 injection, characterized by increased alternation rate after sub-chronic administration ( $P=0.005$ compared to stressed mice). A similar result was obtained in the age-induced cognitive deficit model, where GL-II-73 ( $p=0.0001)$ but not GL-II-75 ( $p=0.46)$ increased alternation rate after sub-chronic administration. 


\section{DISCUSSION}

The present study identified for the first time the combined antidepressant, anxiolytic and procognitive properties of newly designed IBDZ amide ligands with a5-PAM activity. This work is based on the hypotheses that deficits in signaling through these receptors contribute to mood and cognitive symptoms in depression and during aging, and that selective enhancement of activity at a5-GABAA-Rs may have therapeutic potential, compared to non-selective BZs that lack such activities. We generated four ligands; metabolic stability of one of them (GL-II-76) was unfavorable, potentially due to the pyrrolidine residue in the amide moiety, and it was not tested further. Binding and electrophysiological assessment indicated that GL-II-73 and GL-II-74 acted as PAMs with priority affinity and efficacy at a5-GABAA-Rs, whereas GL-II-75 potentiated GABA-gated chloride current to a greater extent at $\alpha 1-$, $\alpha 2-$ and $\alpha 3-$ GABAA-Rs compared to $\alpha 5-$ GABAA-R, suggesting properties closer to DZP (48), although with much lower affinity at $\alpha$ GABAA-Rs than DZP. The lack of potentiation at GABAA-Rs containing the $\alpha 4, \alpha 6$ or $\delta$ subunits (54) supports the notion that all ligands bind to the DZP-specific BZ-sensitive site of GABAA-Rs.

Brain and plasma pharmacokinetic studies showed that the ligands are brain-penetrant, with penetration indices $\left(\mathrm{K}_{\mathrm{p}}, \mathrm{K}_{\mathrm{p}, \mathrm{uu}}\right)$ ranked in the following decreasing order: DZP > GL-II-74 > GL-II$75>$ GL-II-73. Accordingly, all ligands were tested for behavioral activity in vivo. The three newly-designed ligands as well as the non-selective DZP (55) exerted an anxiolytic effect, demonstrating conserved anxiolytic effects despite variable efficacy profiles, probably attributable to a sufficient degree of modulatory activity at $\alpha 2-$ GABAA-Rs (27). In the forcedswim test, all three new ligands displayed antidepressant-like properties, at doses that induced no or low locomotor effects. GL-II-74 and GL-II-75 demonstrated altered locomotor activity and diminished coordination at the higher doses $(10 \mathrm{mg} / \mathrm{kg})$, suggesting motor-impairing side-effects, probably due to their higher potentiation at $\alpha 1-$ GABAA-Rs $(56,57)$. On the other hand, the affinity of GL-II-73 for a1-GABAA-Rs is exceptionally low, which profoundly decreases the 
propensity of this ligand to elicit any motor impairment. In contrast, DZP increased the time spent immobile, confirming a lack of antidepressant effect, or even an induction of depressantlike state, although the latter was probably confounded by motor-impairing effects (58).

We tested the potential of novel ligands at reversing spatial working memory deficits induced by chronic stress exposure(14,59) or related to normal aging $(60,61)$. GL-II-73 and GLII-75 exhibited significant pro-cognitive efficacy in the stress-induced cognitive deficit model, whereas GL-II-74 displayed no pro-cognitive effects. Notably, the pro-cognitive effects of GL-II73 and GL-II-75 were confirmed in aged mice, as old animals performed at levels observed in young animals after a single injection. The pro-cognitive effect of GL-II-73, but not GL-II-75, was maintained after sub-chronic administration in both the stress-induced and aging models. It remains to be tested whether this difference reflected the shorter brain half-life or other kinetic parameters of GL-II-75, or a potential desensitization effect. Consistent with previous findings, DZP had no pro-cognitive effects (62). Together, these results demonstrate for the first time the potential for anxiolytic and combined antidepressant and pro-cognitive properties of newly designed IBDZ amide ligands with efficacy at a5-GABAA-Rs.

It was recently shown that co-localization of the $\alpha 1-, \alpha 5-$ and $y 2-s u b u n i t$ in the hippocampus is necessary for successful spatial learning (26). From this postulate, the potentiation at both a5and a1-GABAA-Rs could explain pro-cognitive properties of GL-II-73 and GL-II-75 after acute/sub-chronic administration in our working memory task, however it does not explain the lack of pro-cognitive effects of GL-II-74 and of BZs in general. Moreover, it was shown in experiments combining DZP with a5- or a1-GABAA-R antagonists that DZP incapacitation action in the Morris water maze, a reference spatial learning and memory test (63), are mediated by a1-GABAAR activation and that blockade of a5-GABAAR activity further worsen this incapacitation.

Could all these apparently disparate findings be reconciled in a consistent way in light of other parameters tested or estimated in this study? We suggest that the relative fraction of 
receptors occupied may be a relevant factor (Table 1). Despite significant efficacy and robust behavioral effects, GL-II-73 displayed unprecedented low overall receptor occupancies, compared to GL-II-74, GL-II-75 and DZP. This suggests that a low fractional occupancy at $\alpha 1$ GABAA-Rs ( $<10 \%$, and preferentially $<1 \%)$, accompanied by a moderate level of positive modulation at both $\alpha 1$ - and a5-GABAA-Rs, gives rise to an improved side-effect profile, together unmasking antidepressant or pro-cognitive potential of a5-GABAA-Rs. Hence, high fractional occupation may preclude any antidepressant or pro-cognitive properties, consistent with the prodepressant-like(55) and amnesic effect of DZP (64) suggested to be mediated by a1GABAA-Rs $(65,66)$. The role of the $\alpha 1$ - and a5-subunit seems to follow a biphasic pattern, where low activation facilitates mood and cognitive processes whereas high and sustained activation impairs these functions (67).

Negative allosteric modulators (NAMs) at GABAA-Rs can also exert antidepressant (68) and pro-cognitive $(69,70)$ activity in tests such as passive avoidance learning, recognition learning (71) and spatial memory (72). Moreover, patients with cognitive impairment in Down syndrome were unsuccessfully clinically-trialed with the a5-selective NAM basmisanil, which is currently being tested for cognitive impairment associated with schizophrenia (73). However, reducing the function of a5-GABAA-Rs is predicted to worsen the pathology associated with reduced SOMATOSTATIN cell function and increased neuronal excitability, hence being potentially associated with risk for long-term detrimental effects, and Alzheimer's disease development (74). However, the putative pro-cognitive properties of both a5-NAMs and a5-PAMs are not mutually incompatible. a5-NAM could be acutely efficient in certain cognitive tasks such as spatial reference (70) where disinhibition of pyramidal neurons may facilitate the acquisition of a mental spatial map. In contrast, an a5-PAM could be efficient in cognitive tasks (such as working memory) where increased inhibition of neuronal activity may reduce noise and interferences, and increase signal-to-noise ratio for incoming stimuli $(6,75)$, together 
strengthening the salient inputs that need to be kept for high-level performances (76). This hypothesis on the roles of NAMs and PAMs will require further validation.

Given the increased rates of cognitive and mood impairments with psychiatric diseases and aging, it is imperative to develop new therapeutic strategies based on emerging knowledge of primary pathologies of the diseases. Here, we designed, developed, tested and validated preclinical efficacies of novel IBZD amide ligands acting either preferentially (GL-II-73 and GL-II74) or non-selectively (GL-II-75) as a5-PAM at GABAA-R, hence bypassing the putative SOMATOSTATIN-cell GABA deficit by acting on post-synaptic sites. The fact that these ligands are synthetized from the hybrid DZP/flumazenil privilege structure with very low toxicity profiles and exhibit combined antidepressant and pro-cognitive therapeutic potential (for GL-II-73 and GL-II-75), suggest they may have clinical potential through simultaneously targeting mood and cognition, therefore reducing cognitive deficits associated with depression and other psychiatric disorders, as well as normal aging. 


\section{ACKNOWLEDGEMENTS}

We acknowledge the University of Wisconsin-Milwaukee's Shimadzu Laboratory for Advanced and Applied Analytical Chemistry, the NIH (R01MH096463: R01NS076517), the Ministry of Education, Science and Technological Development, R. Serbia (Grant No. 175076), and the Brain \& Behavior Resrach Foundation, awarding NARSAD grants (ES, MB) for generous financial support. We thank the Milwaukee Institute of Drug Design.

\section{DISCLOSURES}

ES, JC, MS and MB are co-inventors or listed on a U.S. provisional patent application that covers the described ligands modulating the function of GABA neurons. The other authors report no biomedical financial interests or potential conflicts of interest. 


\section{REFERENCES}

1. Lin LC, Sibille E (2015): Somatostatin, neuronal vulnerability and behavioral emotionality. Molecular psychiatry. 20:377-387.

2. Luscher B, Fuchs T (2015): GABAergic control of depression-related brain states. Advances in pharmacology. 73:97-144.

3. Lewis DA, Hashimoto T, Volk DW (2005): Cortical inhibitory neurons and schizophrenia. Nature reviews Neuroscience. 6:312-324.

4. Nemeroff CB (2003): The role of GABA in the pathophysiology and treatment of anxiety disorders. Psychopharmacology bulletin. 37:133-146.

5. Gavilan MP, Revilla E, Pintado C, Castano A, Vizuete ML, Moreno-Gonzalez I, et al. (2007): Molecular and cellular characterization of the age-related neuroinflammatory processes occurring in normal rat hippocampus: potential relation with the loss of somatostatin GABAergic neurons. Journal of neurochemistry. 103:984-996.

6. Fee C, Banasr M, Sibille E (2017): Somatostatin-Positive Gamma-Aminobutyric Acid Interneuron Deficits in Depression: Cortical Microcircuit and Therapeutic Perspectives. Biological psychiatry.

7. Fuhrer TE, Palpagama TH, Waldvogel HJ, Synek BJL, Turner C, Faull RL, et al. (2017): Impaired expression of GABA transporters in the human Alzheimer's disease hippocampus, subiculum, entorhinal cortex and superior temporal gyrus. Neuroscience. 351:108-118.

8. Rajkowska G, O'Dwyer G, Teleki Z, Stockmeier CA, Miguel-Hidalgo JJ (2007): GABAergic neurons immunoreactive for calcium binding proteins are reduced in the prefrontal cortex in major depression. Neuropsychopharmacology : official publication of the American College of Neuropsychopharmacology. 32:471-482.

9. Price RB, Shungu DC, Mao X, Nestadt P, Kelly C, Collins KA, et al. (2009): Amino acid neurotransmitters assessed by proton magnetic resonance spectroscopy: relationship to treatment resistance in major depressive disorder. Biol Psychiatry. 65:792-800.

10. Guilloux JP, Douillard-Guilloux G, Kota R, Wang X, Gardier AM, Martinowich K, et al. (2012): Molecular evidence for BDNF- and GABA-related dysfunctions in the amygdala of female subjects with major depression. Molecular psychiatry. 17:1130-1142.

11. Prevot TD, Gastambide F, Viollet C, Henkous N, Martel G, Epelbaum J, et al. (2017): Roles of Hippocampal Somatostatin Receptor Subtypes in Stress Response and Emotionality. Neuropsychopharmacology : official publication of the American College of Neuropsychopharmacology. 42:1647-1656.

12. Soumier A, Sibille E (2014): Opposing effects of acute versus chronic blockade of frontal cortex somatostatin-positive inhibitory neurons on behavioral emotionality in mice. Neuropsychopharmacology : official publication of the American College of Neuropsychopharmacology. 39:2252-2262.

13. Fuchs T, Jefferson SJ, Hooper A, Yee PH, Maguire J, Luscher B (2016): Disinhibition of somatostatin-positive GABAergic interneurons results in an anxiolytic and antidepressant-like brain state. Molecular psychiatry.

14. Prevot TD, Viollet C, Epelbaum J, Dominguez G, Beracochea D, Guillou JL (2018): sst2-receptor gene deletion exacerbates chronic stress-induced deficits: Consequences for emotional and cognitive ageing. Progress in neuro-psychopharmacology \& biological psychiatry.

15. Epelbaum J, Guillou JL, Gastambide F, Hoyer D, Duron E, Viollet C (2009): Somatostatin, Alzheimer's disease and cognition: an old story coming of age? Progress in neurobiology. 89:153-161. 
16. Schmid LC, Mittag M, Poll S, Steffen J, Wagner J, Geis HR, et al. (2016): Dysfunction of Somatostatin-Positive Interneurons Associated with Memory Deficits in an Alzheimer's Disease Model. Neuron. 92:114-125.

17. Mohler H (2006): GABA(A) receptor diversity and pharmacology. Cell and tissue research. 326:505-516.

18. Ali AB, Thomson AM (2008): Synaptic alpha 5 subunit-containing GABAA receptors mediate IPSPs elicited by dendrite-preferring cells in rat neocortex. Cerebral cortex. 18:1260-1271.

19. Botta P, Demmou L, Kasugai Y, Markovic M, Xu C, Fadok JP, et al. (2015): Regulating anxiety with extrasynaptic inhibition. Nature neuroscience. 18:1493-1500.

20. Brickley SG, Mody I (2012): Extrasynaptic GABA(A) receptors: their function in the CNS and implications for disease. Neuron. 73:23-34.

21. Palmer L, Murayama M, Larkum M (2012): Inhibitory Regulation of Dendritic Activity in vivo. Frontiers in neural circuits. 6:26.

22. Dunlop BW, Davis PG (2008): Combination treatment with benzodiazepines and SSRIs for comorbid anxiety and depression: a review. Prim Care Companion J Clin Psychiatry. 10:222-228.

23. Parker GB, Graham RK (2015): Determinants of Treatment-Resistant Depression: The Salience of Benzodiazepines. The Journal of nervous and mental disease. 203:659-663.

24. Sigel E, Ernst M (2018): The Benzodiazepine Binding Sites of GABAA Receptors. Trends in pharmacological sciences.

25. Mohler H (2015): The legacy of the benzodiazepine receptor: from flumazenil to enhancing cognition in Down syndrome and social interaction in autism. Advances in pharmacology. 72:1-36.

26. Ghafari M, Falsafi SK, Szodorai E, Kim EJ, Li L, Hoger H, et al. (2017): Formation of GABAA receptor complexes containing alpha1 and alpha5 subunits is paralleling a multiple T-maze learning task in mice. Brain structure \& function. 222:549-561.

27. Smith KS, Engin E, Meloni EG, Rudolph U (2012): Benzodiazepine-induced anxiolysis and reduction of conditioned fear are mediated by distinct GABAA receptor subtypes in mice. Neuropharmacology. 63:250-258.

28. Hortnagl H, Tasan RO, Wieselthaler A, Kirchmair E, Sieghart W, Sperk G (2013): Patterns of mRNA and protein expression for 12 GABAA receptor subunits in the mouse brain. Neuroscience. 236:345-372.

29. Gill KM, Grace AA (2014): The role of alpha5 GABAA receptor agonists in the treatment of cognitive deficits in schizophrenia. Current pharmaceutical design. 20:5069-5076.

30. Behlke LM, Foster RA, Liu J, Benke D, Benham RS, Nathanson AJ, et al. (2016): A Pharmacogenetic 'Restriction-of-Function' Approach Reveals Evidence for Anxiolytic-Like Actions Mediated by alpha5-Containing GABAA Receptors in Mice. Neuropsychopharmacology : official publication of the American College of Neuropsychopharmacology. 41:2492-2501.

31. Gill KM, Lodge DJ, Cook JM, Aras S, Grace AA (2011): A Novel a5GABA(A)R-Positive Allosteric Modulator Reverses Hyperactivation of the Dopamine System in the MAM Model of Schizophrenia. Neuropsychopharmacology. 36:1903-1911.

32. Gill KM, Cook JM, Poe MM, Grace AA (2014): Prior Antipsychotic Drug Treatment Prevents Response to Novel Antipsychotic Agent in the Methylazoxymethanol Acetate Model of Schizophrenia. Schizophr Bull. 40:341-350.

33. Piantadosi SC, French BJ, Poe MM, Timic T, Markovic BD, Pabba M, et al. (2016): Sex-Dependent Anti-Stress Effect of an alpha5 Subunit Containing GABAA Receptor Positive Allosteric Modulator. Frontiers in pharmacology. 7:446.

34. Koh MT, Rosenzweig-Lipson S, Gallagher M (2013): Selective GABA(A) alpha5 positive allosteric modulators improve cognitive function in aged rats with memory impairment. Neuropharmacology. 64:145-152. 
35. Clayton T, Chen JL, Ernst M, Richter L, Cromer BA, Morton CJ, et al. (2007): An updated unified pharmacophore model of the benzodiazepine binding site on gamma-aminobutyric acid(a) receptors: correlation with comparative models. Curr Med Chem. 14:2755-2775.

36. Savic MM, Clayton T, Furtmuller R, Gavrilovic I, Samardzic J, Savic S, et al. (2008): PWZ-029, a compound with moderate inverse agonist functional selectivity at GABA(A) receptors containing alpha5 subunits, improves passive, but not active, avoidance learning in rats. Brain Res. 1208:150-159.

37. Clayton T, Poe MM, Rallapalli S, Biawat P, Savic MM, Rowlett JK, et al. (2015): A Review of the Updated Pharmacophore for the Alpha 5 GABA(A) Benzodiazepine Receptor Model. Int J Med Chem. 2015:430248.

38. Namjoshi OA, Wang Z-j, Rallapalli SK, Johnson EM, Johnson Y-T, Ng H, et al. (2013): Search for $\alpha 3 \beta(2 / 3) \gamma 2$ Subtype Selective Ligands That are Stable on Human Liver Microsomes. Bioorg Med Chem. 21:93-101.

39. Stamenic TT, Poe MM, Rehman S, Santrac A, Divovic B, Scholze P, et al. (2016): Ester to amide substitution improves selectivity, efficacy and kinetic behavior of a benzodiazepine positive modulator of GABAA receptors containing the alpha5 subunit. European journal of pharmacology. 791:433-443.

40. Richetto J, Labouesse MA, Poe MM, Cook JM, Grace AA, Riva MA, et al. (2015): Behavioral effects of the benzodiazepine-positive allosteric modulator SH-053-2'F-S-CH(3) in an immune-mediated neurodevelopmental disruption model. The international journal of neuropsychopharmacology. 18.

41. Li G, Stephen MR, Kodali R, Zahn NM, Poe MM, Tiruveedhula VVNPB, et al. (2018): Synthesis of chiral GABAA receptor subtype selective ligands as potential agents to treat schizophrenia as well as depression. ARKIVOC.

42. Alexeev M, Grosenbaugh DK, Mott DD, Fisher JL (2012): The natural products magnolol and honokiol are positive allosteric modulators of both synaptic and extra-synaptic GABA(A) receptors. Neuropharmacology. 62:2507-2514.

43. Cheng Y, Prusoff WH (1973): Relationship between the inhibition constant (K1) and the concentration of inhibitor which causes 50 per cent inhibition (I50) of an enzymatic reaction. Biochemical pharmacology. 22:3099-3108.

44. Ghosal S, Nunley A, Mahbod P, Lewis AG, Smith EP, Tong J, et al. (2015): Mouse handling limits the impact of stress on metabolic endpoints. Physiology \& behavior. 150:31-37.

45. Dalvi A, Rodgers RJ (2001): Anxiolytic effects of valproate and diazepam in mice are differentially sensitive to picrotoxin antagonism. Pharmacology, biochemistry, and behavior. 68:23-32.

46. Namjoshi OA, Wang ZJ, Rallapalli SK, Johnson EM, Jr., Johnson YT, Ng H, et al. (2013): Search for alpha3beta(2)/(3)gamma2 subtype selective ligands that are stable on human liver microsomes. Bioorganic \& medicinal chemistry. 21:93-101.

47. Obradovic A, Joksimovic S, Poe MM, Ramerstorfer J, Varagic Z, Namjoshi O, et al. (2014): Sh-I048A, an in vitro non-selective super-agonist at the benzodiazepine site of GABAA receptors: the approximated activation of receptor subtypes may explain behavioral effects. Brain research. 1554:3648.

48. Lewter LA, Fisher JL, Siemian JN, Methuku KR, Poe MM, Cook JM, et al. (2017): Antinociceptive Effects of a Novel alpha2/alpha3-Subtype Selective GABAA Receptor Positive Allosteric Modulator. ACS chemical neuroscience. 8:1305-1312.

49. Savic MM, Majumder S, Huang SM, Edwankar RV, Furtmuller R, Joksimovic S, et al. (2010): Novel positive allosteric modulators of GABAA receptors: Do subtle differences in activity at alpha 1 plus alpha 5 versus alpha 2 plus alpha 3 subunits account for dissimilarities in behavioral effects in rats? Progress in neuro-psychopharmacology \& biological psychiatry. 34:376-386.

50. Hadingham KL, Garrett EM, Wafford KA, Bain C, Heavens RP, Sirinathsinghji DJ, et al. (1996): Cloning of cDNAs encoding the human gamma-aminobutyric acid type A receptor alpha 6 subunit and 
characterization of the pharmacology of alpha 6-containing receptors. Molecular pharmacology. 49:253259.

51. Wafford KA, Thompson SA, Thomas D, Sikela J, Wilcox AS, Whiting PJ (1996): Functional characterization of human gamma-aminobutyric acidA receptors containing the alpha 4 subunit. Molecular pharmacology. 50:670-678.

52. Wisden W, Herb A, Wieland H, Keinanen K, Luddens H, Seeburg PH (1991): Cloning, pharmacological characteristics and expression pattern of the rat GABAA receptor alpha 4 subunit. FEBS letters. 289:227-230.

53. Gustafsson S, Lindstrom V, Ingelsson M, Hammarlund-Udenaes M, Syvanen S (2018): Intact blood-brain barrier transport of small molecular drugs in animal models of amyloid beta and alphasynuclein pathology. Neuropharmacology. 128:482-491.

54. Gunther U, Benson J, Benke D, Fritschy JM, Reyes G, Knoflach F, et al. (1995): Benzodiazepineinsensitive mice generated by targeted disruption of the gamma 2 subunit gene of gamma-aminobutyric acid type A receptors. Proceedings of the National Academy of Sciences of the United States of America. 92:7749-7753.

55. Flugy A, Gagliano M, Cannizzaro C, Novara V, Cannizzaro G (1992): Antidepressant and anxiolytic effects of alprazolam versus the conventional antidepressant desipramine and the anxiolytic diazepam in the forced swim test in rats. European journal of pharmacology. 214:233-238.

56. McKernan RM, Rosahl TW, Reynolds DS, Sur C, Wafford KA, Atack JR, et al. (2000): Sedative but not anxiolytic properties of benzodiazepines are mediated by the GABA(A) receptor alpha1 subtype. Nature neuroscience. 3:587-592.

57. Korttila K, Linnoila M (1975): Recovery and skills related to driving after intravenous sedation: dose-response relationship with diazepam. British journal of anaesthesia. 47:457-463.

58. El Zahaf NA, Elhwuegi AS (2014): The effect of GABAmimetics on the duration of immobility in the forced swim test in albino mice. The Libyan journal of medicine. 9:23480.

59. Minni AM, Dorey R, Pierard C, Dominguez G, Helbling JC, Foury A, et al. (2012): Critical role of plasma corticosteroid-binding-globulin during stress to promote glucocorticoid delivery to the brain: impact on memory retrieval. Endocrinology. 153:4766-4774.

60. Vandesquille M, Krazem A, Louis C, Lestage P, Beracochea D (2011): S 18986 reverses spatial working memory impairments in aged mice: comparison with memantine. Psychopharmacology. 215:709-720.

61. Vandesquille M, Baudonnat M, Decorte L, Louis C, Lestage P, Beracochea D (2013): Working memory deficits and related disinhibition of the CAMP/PKA/CREB are alleviated by prefrontal alpha4beta2*-nAChRs stimulation in aged mice. Neurobiology of aging. 34:1599-1609.

62. Griffin CE, 3rd, Kaye AM, Bueno FR, Kaye AD (2013): Benzodiazepine pharmacology and central nervous system-mediated effects. The Ochsner journal. 13:214-223.

63. Savic MM, Milinkovic MM, Rallapalli S, Clayton T, Sr., Joksimovic S, Van Linn M, et al. (2009): The differential role of alpha1- and alpha5-containing GABA(A) receptors in mediating diazepam effects on spontaneous locomotor activity and water-maze learning and memory in rats. The international journal of neuropsychopharmacology. 12:1179-1193.

64. Lister RG (1985): The amnesic action of benzodiazepines in man. Neuroscience and biobehavioral reviews. 9:87-94.

65. Rudolph U, Crestani F, Benke D, Brunig I, Benson JA, Fritschy JM, et al. (1999): Benzodiazepine actions mediated by specific gamma-aminobutyric acid(A) receptor subtypes. Nature. 401:796-800.

66. Savic MM, Obradovic DI, Ugresic ND, Cook JM, Yin W, Bokonjic DR (2005): Bidirectional effects of benzodiazepine binding site ligands in the passive avoidance task: differential antagonism by flumazenil and beta-CCt. Behavioural brain research. 158:293-300. 
67. Zurek AA, Yu J, Wang DS, Haffey SC, Bridgwater EM, Penna A, et al. (2014): Sustained increase in alpha5GABAA receptor function impairs memory after anesthesia. The Journal of clinical investigation. 124:5437-5441.

68. Zanos P, Nelson ME, Highland JN, Krimmel SR, Georgiou P, Gould TD, et al. (2017): A Negative Allosteric Modulator for alpha5 Subunit-Containing GABA Receptors Exerts a Rapid and Persistent Antidepressant-Like Action without the Side Effects of the NMDA Receptor Antagonist Ketamine in Mice. eNeuro. 4.

69. Collinson N, Kuenzi FM, Jarolimek W, Maubach KA, Cothliff R, Sur C, et al. (2002): Enhanced learning and memory and altered GABAergic synaptic transmission in mice lacking the alpha 5 subunit of the GABAA receptor. The Journal of neuroscience : the official journal of the Society for Neuroscience. 22:5572-5580.

70. Braudeau J, Delatour B, Duchon A, Pereira PL, Dauphinot L, de Chaumont F, et al. (2011): Specific targeting of the GABA-A receptor alpha5 subtype by a selective inverse agonist restores cognitive deficits in Down syndrome mice. Journal of psychopharmacology. 25:1030-1042.

71. Milic M, Timic T, Joksimovic S, Biawat P, Rallapalli S, Divljakovic J, et al. (2013): PWZ-029, an inverse agonist selective for alpha(5) GABAA receptors, improves object recognition, but not watermaze memory in normal and scopolamine-treated rats. Behavioural brain research. 241:206-213.

72. Martinez-Cue C, Martinez P, Rueda N, Vidal R, Garcia S, Vidal V, et al. (2013): Reducing GABAA alpha5 receptor-mediated inhibition rescues functional and neuromorphological deficits in a mouse model of down syndrome. The Journal of neuroscience : the official journal of the Society for Neuroscience. 33:3953-3966.

73. Roche H-L (2016): A Study to Evaluate the Effects of Basmisanil in Participants With Cognitive Impairment Associated With Schizophrenia (CIAS) Treated With Antipsychotics. ClinicalTrialsgov.

74. Verret L, Mann EO, Hang GB, Barth AM, Cobos I, Ho K, et al. (2012): Inhibitory interneuron deficit links altered network activity and cognitive dysfunction in Alzheimer model. Cell. 149:708-721.

75. Murray JD, Anticevic A, Gancsos M, Ichinose M, Corlett PR, Krystal JH, et al. (2014): Linking microcircuit dysfunction to cognitive impairment: effects of disinhibition associated with schizophrenia in a cortical working memory model. Cerebral cortex. 24:859-872.

76. Mohler H, Rudolph U (2017): Disinhibition, an emerging pharmacology of learning and memory. F1000Research. 6. 


\section{Figure 1. Electrophysiological recordings at $\mathrm{GABA}_{A}$ receptors}

Modulation properties of 100nM or $1 \mu \mathrm{M}$ of GL-II-73 (A,E), GL-II-74 (B,F), GL-II-75 (C,G) or GLII-76 $(\mathbf{D}, \mathbf{H})$ at recombinant $\alpha 1 / 2 / 3 / 4 / 5 / 6 \beta 3 y 2$ (a-d) or $\alpha 1 \beta 1 / 3 y 2$ (e-h) receptors. The potentiation of GABA alone (EC $\left.\mathrm{EC}_{3-5} \mathrm{GABA}\right)$ is expressed in percentage. ${ }^{*} \mathrm{p}<0.05 ;{ }^{* *} \mathrm{p}<0.01$ and ${ }^{* * *} \mathrm{p}<0.001$ compared to $100 \%, \# p<0.05$ compared to $\alpha 1 \beta 3 y 2$. The approximated electrophysiological responses elicited by the estimated free brain concentrations and presented on the concentration-response curves of GL-II-73 (I), GL-II-74 (J), GL-II-75 (K) and DZP (L) at rat recombinant $\alpha 1 \beta 3 \gamma 2$ and $\alpha 5 \beta 3 y 2$ GABAA receptors measured at GABA EC3 (eliciting $3 \%$ of the maximal GABA current in the respective subtype). Brain tissue density of $1.04 \mathrm{~g} / \mathrm{ml}$ was used to convert brain concentrations from $\mathrm{ng} / \mathrm{g}$ into $\mathrm{ng} / \mathrm{ml}$. The shaded range delineates the interpolated lower and upper limit of potentiation at $a 5 G A B A_{A} R s$ in the dose range used; the vertical lines mark the approximated free brain concentration of the given dose of each ligand. The level of potentiation of $120 \%$ was arbitrarily set as borderline for eliciting in vivo behavioral effects. 
Figure 2. Plasma and brain concentration-time profiles of GL-II-73, GL-II-74, GL-II-75 and DZP

Plasma and brain concentration-time profiles of GL-II-73, GL-II-74, GL-II-75 and DZP after intraperitoneal administration of the $10 \mathrm{mg} / \mathrm{kg}$ dose in male C57BL/6 mice ( $\mathrm{n}=3$ per time point). $\mathrm{C}_{\max }=$ maximum concentration in plasma or brain; $\mathrm{T}_{\max }=$ time of maximum concentration in plasma or brain; $\mathrm{AUC}_{0-720}=$ area under the plasma or brain concentration-time curve from 0 to $720 \mathrm{~min} ; \mathrm{AUC}_{0-\infty}=$ area under the plasma or brain concentration-time curve from 0 to extrapolated infinite time; $t_{1 / 2}=$ elimination half-life from plasma or brain; $\beta=$ elimination constant rate from plasma or brain; $K_{p}=$ brain-to-plasma partition coefficient $\left(K_{p}=A \cup C_{0-\infty}\right.$, brain $/ A C_{0-\infty}$, plasma $) ; \mathrm{K}_{\mathrm{p}, \mathrm{uu} \text {,brain }}=$ ratio of unbound brain to unbound plasma drug concentrations $\left(\mathrm{K}_{\mathrm{p}, \mathrm{uu}, \mathrm{brain}}=\right.$ $\mathrm{Kp} \times$ unbound fraction in brain/unbound fraction in plasma). All values are represented as mean \pm standard error of the mean. 


\section{Figure 3. Anxiolytic and antidepressant properties of the $\alpha-P A M$ compared to diazepam}

Potential anxiolytic action of the new IBZD amide ligands GL-II-73 (A), GL-II-74 (B) and GL-II-75

(C) at 5 or $10 \mathrm{mg} / \mathrm{kg}$ and the referent non-selective PAM diazepam (DZP: $1.5 \mathrm{mg} / \mathrm{kg}$; D) has been assessed in mice (50\% females) using the elevated plus maze. Animals received vehicle or the ligand GL-II-73 $(n(0)=13, n(5)=13$ and $n(10)=14), \quad G L-I I-74 \quad(n(0)=14, n(5)=13$ and $n(10)=14)$, GL-II-75 $(n(0)=13, n(5)=14$ and $n(10)=13)$ or DZP $(n(0)=11$ and $n(1.5)=10), 30$ minutes before testing. A significant increase in the time spent in the open arms was used as an index of potential anxiolytic action. Potential antidepressant properties of the ligands were assessed in male mice using the forced-swim test (E-H). Sex as a cofactor was not significant ( $p$-values $\geq 0.17$ ). Mice were placed in an inescapable transparent tank filled with water $(25 \mathrm{~cm}$, $26 \pm 1^{\circ} \mathrm{C}$ ) for a period of $6 \mathrm{~min}$. Immobility is defined as the minimum amount of movement to stay afloat, between the second and the sixth minute of testing. Mice were injected following serial i.p. administrations at 1,5 or $10 \mathrm{mg} / \mathrm{kg}$ for the new ligands (GL-II-73: $\mathrm{n}(0)=8, \mathrm{n}(1)=8 ; \mathrm{n}(5)=6$ and $n(10)=8 ;$ GL-II-74: $n(0)=8, n(1)=8, n(5)=8$ and $n(10)=8 ; G L-I I-75: n(0)=8, n(1)=8, n(5)=8$ and $n(10)=9)$ or $1.5 \mathrm{mg} / \mathrm{kg}$ for the DZP $(n(0)=12$ and $n(1.5)=12)$. Significant decreased immobility characterized the potential antidepressant-like efficacy of the ligand. ${ }^{* *} p<0.01$ and ${ }^{* *} p<0.001$ compared to Vehicle, \#\#\#p < 0.001 compared to GL-II-73. All values are represented as mean \pm standard error of the mean. 
Figure 4. Pro-cognitive action of GL-II-73 and GL-II-75 on stress-induced and age-related working memory impairment

Cognitive effects were assessed in a spontaneous alternation task using a 90sec inter-trial interval (ITI). Prior to experiment with young mice, a cognitive deficit was induced by exposing the animals to daily chronic restraint stress, 1 hour twice a day for 1 week. Young mice $(50 \%$ females) received vehicle or 1,5 or $10 \mathrm{mg} / \mathrm{kg}$ of $\mathrm{GL}-\mathrm{II}-73(\mathbf{A} ; \mathrm{n}(0-\mathrm{NS})=10, \mathrm{n}(0-\mathrm{S})=10, \mathrm{n}(1)=5$, $n(5)=10, n(10)=12)$. The same protocol was used for $G L-I I-74(B ; n(0-N S)=8, n(0-S)=8, n(1)=4$, $\mathrm{n}(5)=10, \mathrm{n}(10)=4)), \mathrm{GL}-I I-75(\mathbf{C} ; \mathrm{n}(0-\mathrm{NS})=8, \mathrm{n}(0-\mathrm{S})=8, \mathrm{n}(1)=6, \mathrm{n}(5)=4, \mathrm{n}(10)=9)$ and DZP (D; $\mathrm{n}(0-\mathrm{NS})=6, \mathrm{n}(0-\mathrm{S})=6, \mathrm{n}(1,5)=6)$. Animals were injected i.p. with vehicle, a5-PAMs or DZP, 30min prior testing. For old animals (E-F), the same protocol was applied with the ITI shortened to 60sec. Old male mice received Vehicle or GL-II-73 (E; $\mathrm{n}(0-$ Young $)=5, \mathrm{n}(0-\mathrm{Old})=5, \mathrm{n}(5)=6)$ or GL-II-75 (F; n(0-Young)=5, $\mathrm{n}(0-\mathrm{Old})=5, \mathrm{n}(5)=4)$ and were compared to young and old mice treated with Vehicle. For all experiments described here, sex as a cofactor was not significant ( $p$-value $\geq 0.49)$. Results are presented as mean of the percentage of alternation \pm SEM: effect of the stress: ${ }^{*} p<0.05 ;{ }^{* *} p<0.01 ;{ }^{* * *} p<0.001$ compared to "No stress-vehicle"; Effect of age: $++p<0.01$ or $+++p<0.001$ compared to "Young-Vehicle"; Effect of the ligand $\$ p<0.05, \$ \$ p<0.01$ compared to "CRS-Vehicle" or "Old Vehicle". 
Figure 5. Pro-cognitive action of sub-chronically administered GL-II-73 and GL-II-75 on stress-induced and age-related working memory impairment

Pro-cognitive properties of GL-II-73 (A, C) and GL-II-75 (B, D) were assessed in young (A-B) and old (C-D) male mice after sub-chronic administration in the drinking water for 10 days. Cognitive abilities were assessed in a spontaneous alternation task. Alternation rate was calculated as the percentage of correct alternations in function of the maximum alternation possible (i.e. 6). Prior to experiment, a cognitive deficit was induced by exposing the young animals to chronic stress for 1 week. Young animals received sub-chronically GL-II-73 ( $(0$ $N S)=6, n(0-S)=5, n(30)=6)$ or $G L-I I-75(n(0-N S)=5, n(0-S)=5, n(5)=6)$ dosed at 30mg $/ \mathrm{kg}$. Old mice received sub-chronically GL-II-73 $(\mathrm{n}(0-$ Young $)=5, \mathrm{n}(0-\mathrm{Old})=6, \mathrm{n}(30)=4)$ or GL-II-75 $(\mathrm{n}(0$ Young $)=6, \mathrm{n}(0-\mathrm{Old})=5, \mathrm{n}(30)=5)$ dosed at $30 \mathrm{mg} / \mathrm{kg}$ in the drinking water. Results are presented as mean of the percentage of alternation \pm SEM: effect of the stress: ${ }^{*} p<0.05 ;{ }^{* *} p<0.01$; ${ }^{* * *} p<0.001$ compared to "No stress-vehicle"; Effect of age: $++p<0.01$ or $+++p<0.001$ compared to "Young-Vehicle"; Effect of the ligand $\$ p<0.05, \$ \$ p<0.01$ compared to "CS-Vehicle" or "Old Vehicle". 

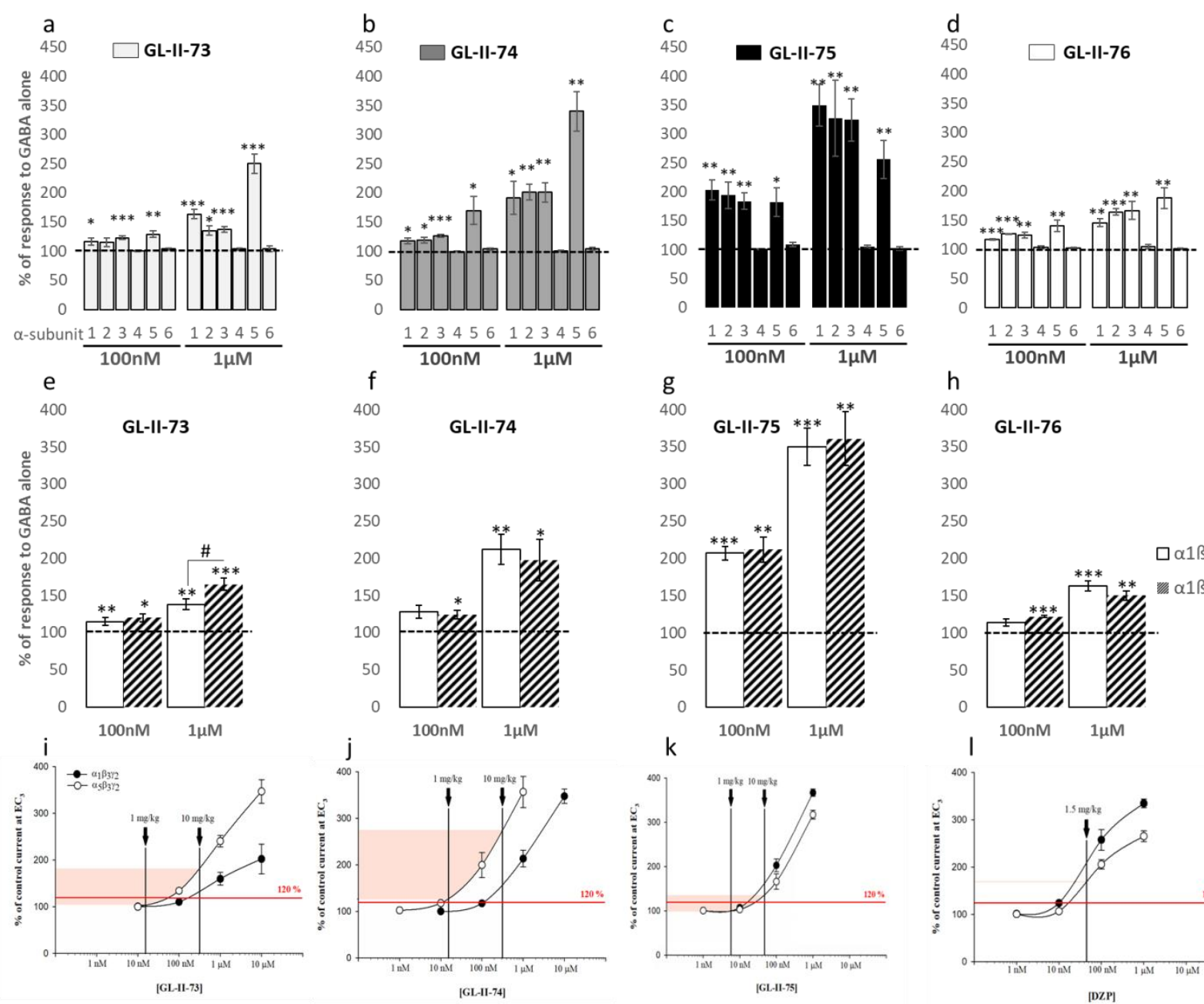

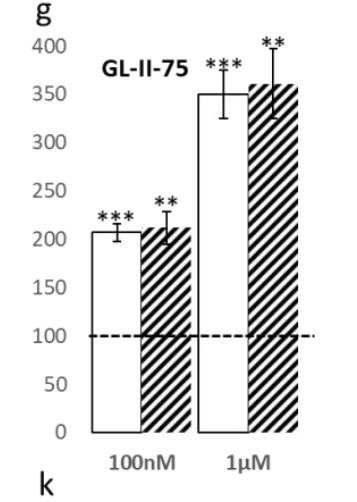

h

400 GL-II-76

350

300
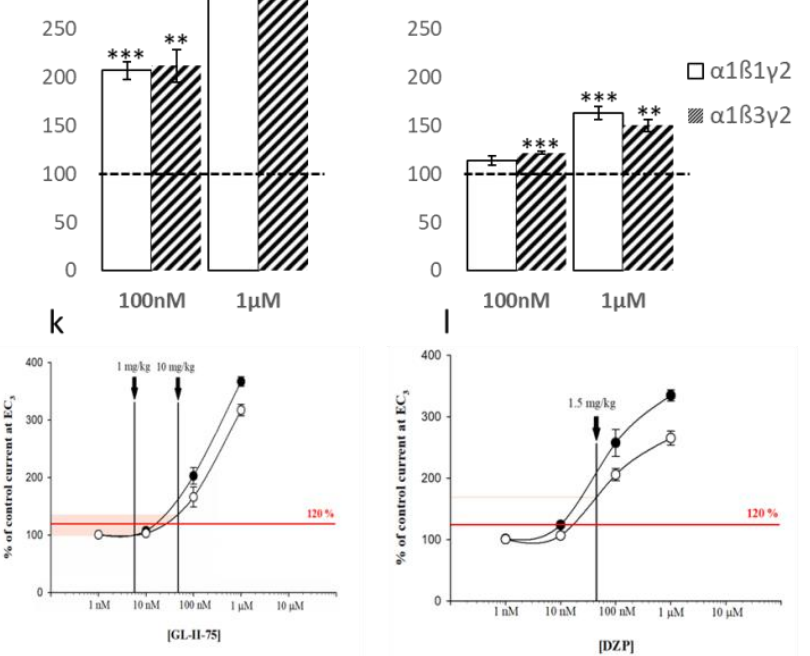

Fig.1 

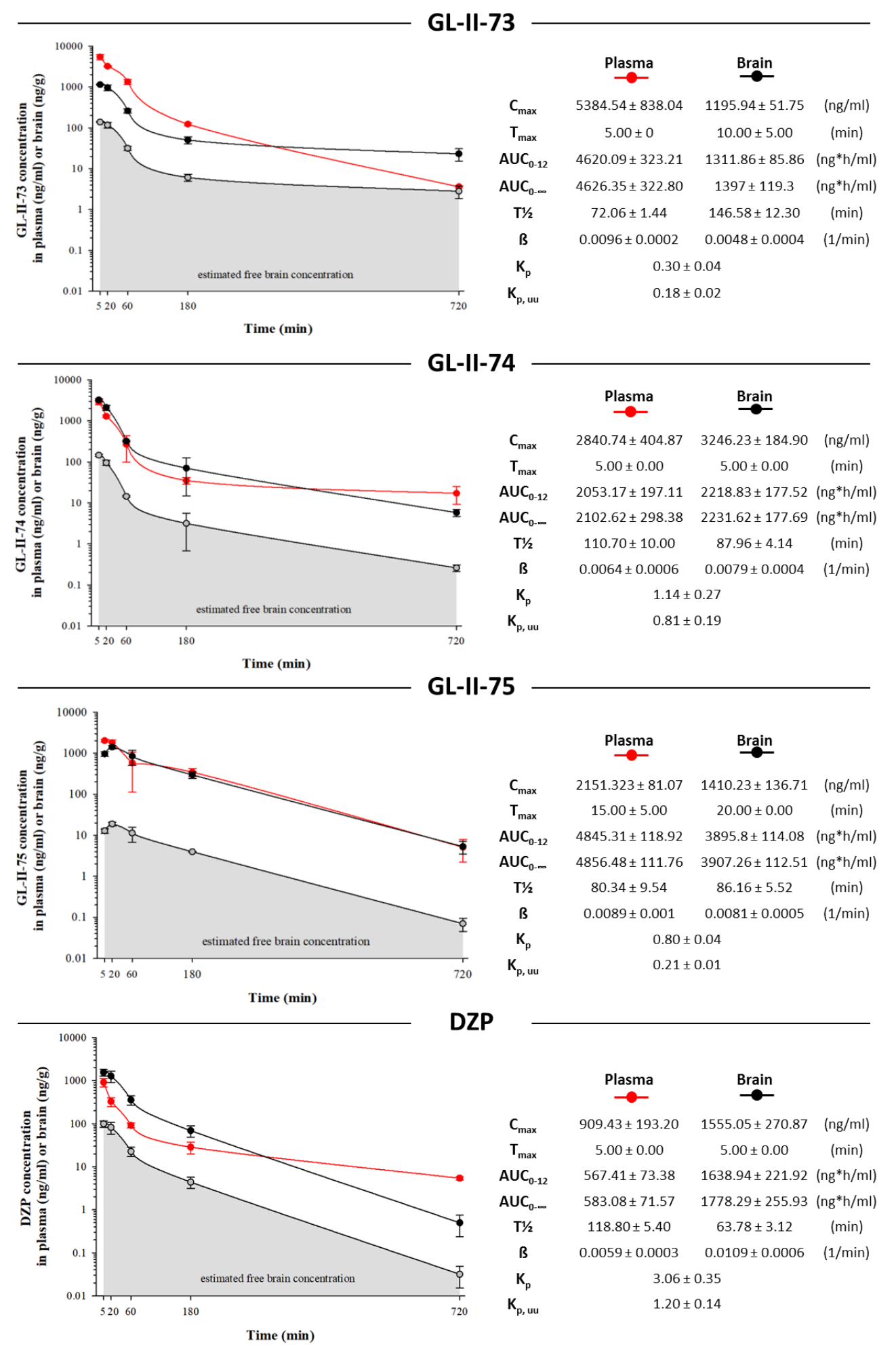

ZP

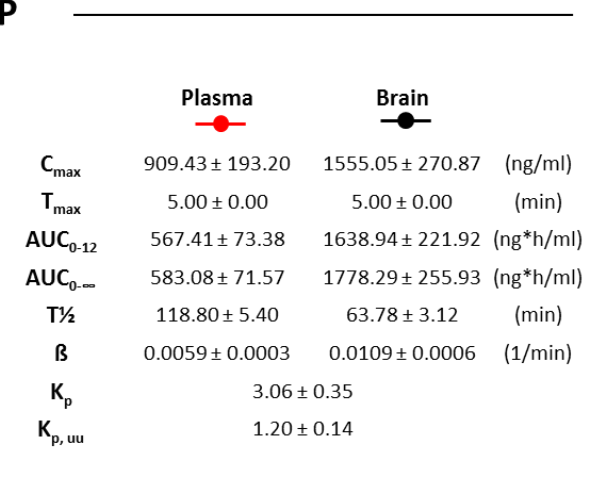

Fig.2 


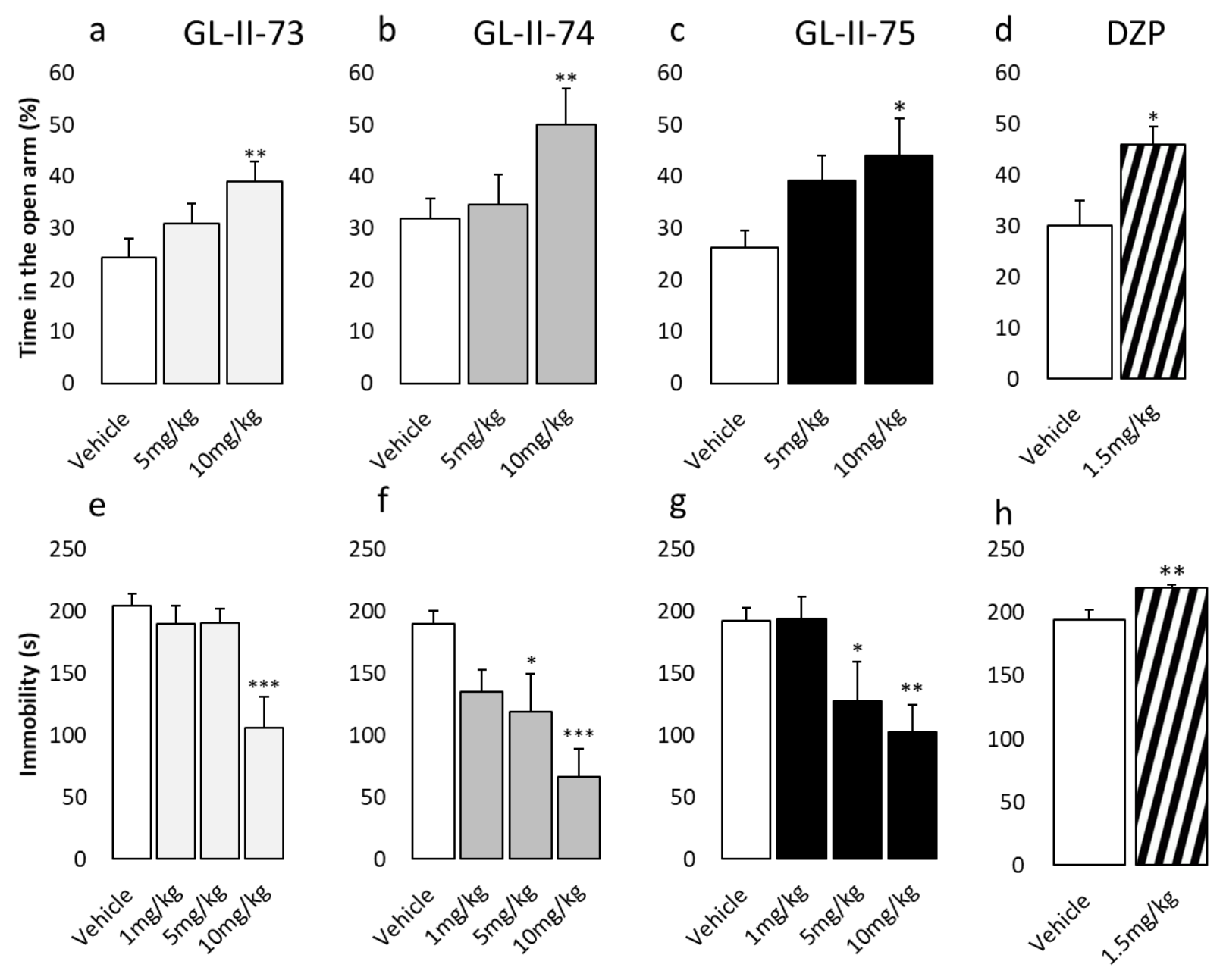

Fig.3 

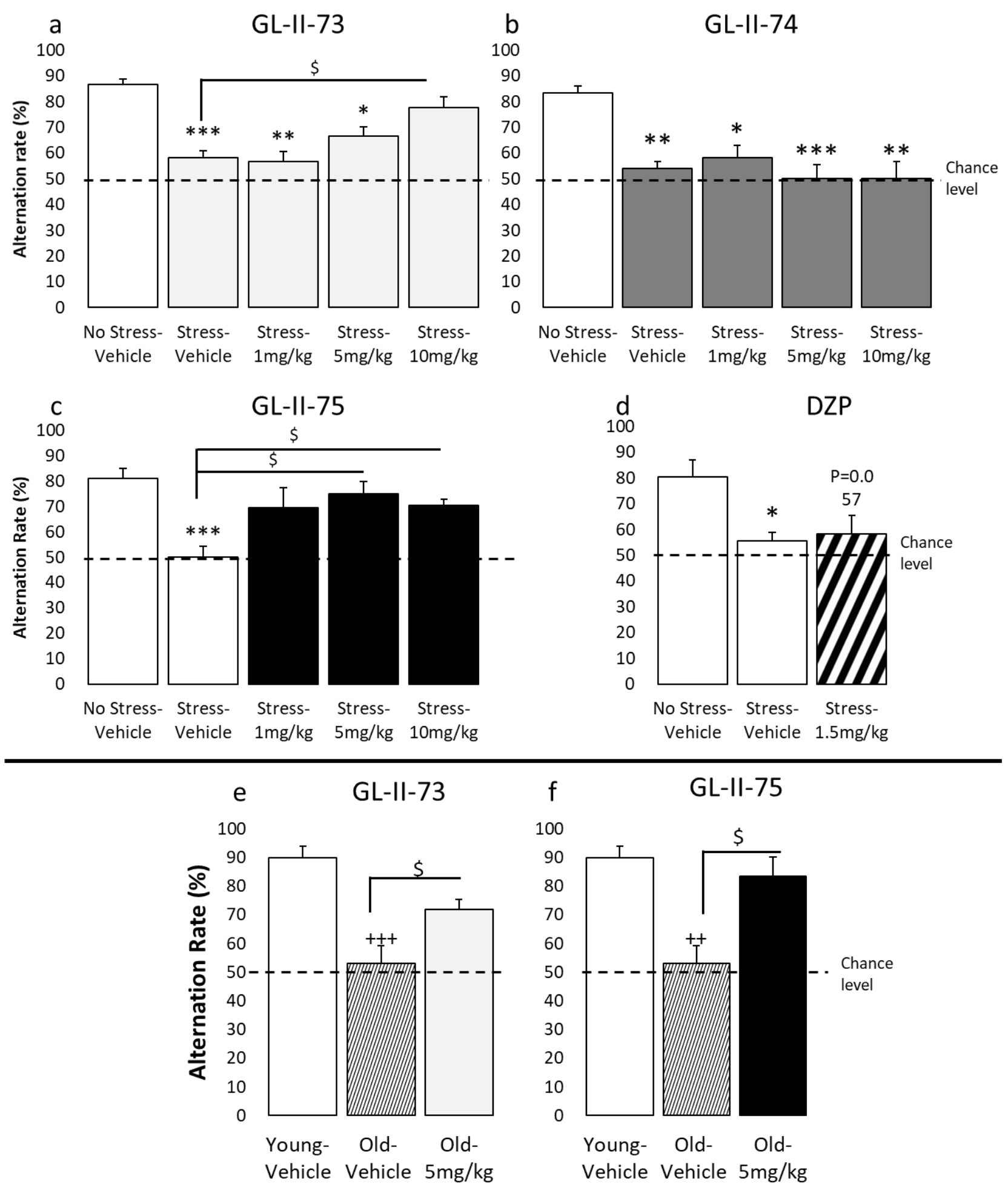

Fig.4 


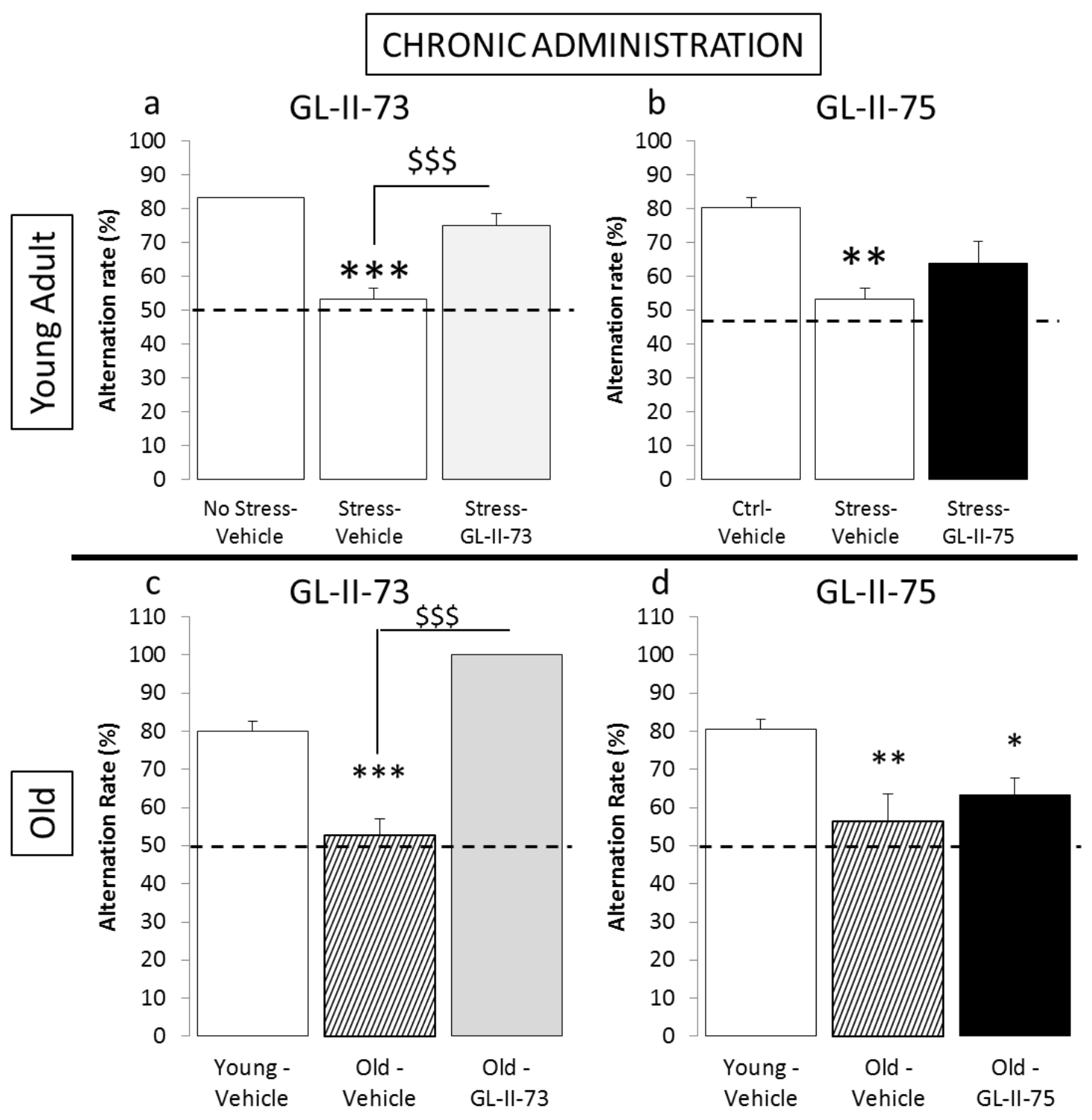

Fig.5 